\section{JTI}

JOURNAL OF

TRAUMA AND INJURY

\title{
Bilateral Asymmetric Traumatic Dislocation of the Hip Joint
}

\author{
Hee Gon Park, M.D., Hyung Suk Yi, M.D., Kyoo Hong Han, M.D. \\ Department of Orthopaedic Surgery, Dankook University College of Medicine, Cheonan, Korea
}

Received: September 25, 2017

Revised: October 22, 2017

Accepted: October 25, 2017

\section{Correspondence to}

\section{Hee Gon Park, M.D.}

Department of Orthopaedic Surgery, Dankook University College of Medicine, 119 Dandae-ro, Dongnam-gu, Cheonan 31116, Korea

Tel: $+82-41-550-6579$

Fax: +82-41-556-3238

E-mail: osdku@dankook.ac.kr

\section{INTRODUCTION}

Traumatic hip joint dislocations account for 2-5\% of total joint dislocations. According to Agarwal et al. [1], bilateral dislocation of hip joint occurs rarely only $1.25 \%$ of the whole hip joint dislocation. Bilateral asymmetric hip joint dislocation with anterior and posterior displacement is an even more rare case because it was according to Loupasis et al. [2] reported in 11 cases only since 1879.

We want to report 2 cases of bilateral asymmetric hip joint dislocation with unilateral acetabular fracture which happened in our hospital. Consent for publication has been obtained from the patients. 


\section{CASE REPORT}

\section{Case 1}

A 53 years old male has been working in an excavator which was shaking due to its weak ground position. The patient fell down from 2 meter height to an obliquely placed rock and rolled several times. He finally came to trauma center of the Dankook university hospital.

Consciousness of the patient was fine based on the 15 Glasgow Coma Scale (GCS) score at emergency room (ER) and multiple abrasions on limbs and chest were observed. The 4th lumbar left transverse process fracture was conservatively treated and there were no other organ trauma or abnormal findings with regard to his hemodynamic stability. His right leg was in abducted, externally rotated and flexed position. The left leg was in adducted, internally rotated and flexed position. Also shortness of left leg was seen with naked eyes.

In the physical examination, blood flow and sensation of both distal legs were normal. The muscle strength of tibialis anterior muscle, extensor hallucis tendon and flexor digitorum marked grade 5 , but we couldn't check psoas and quadriceps muscle power due to severe pain around hip joint area.

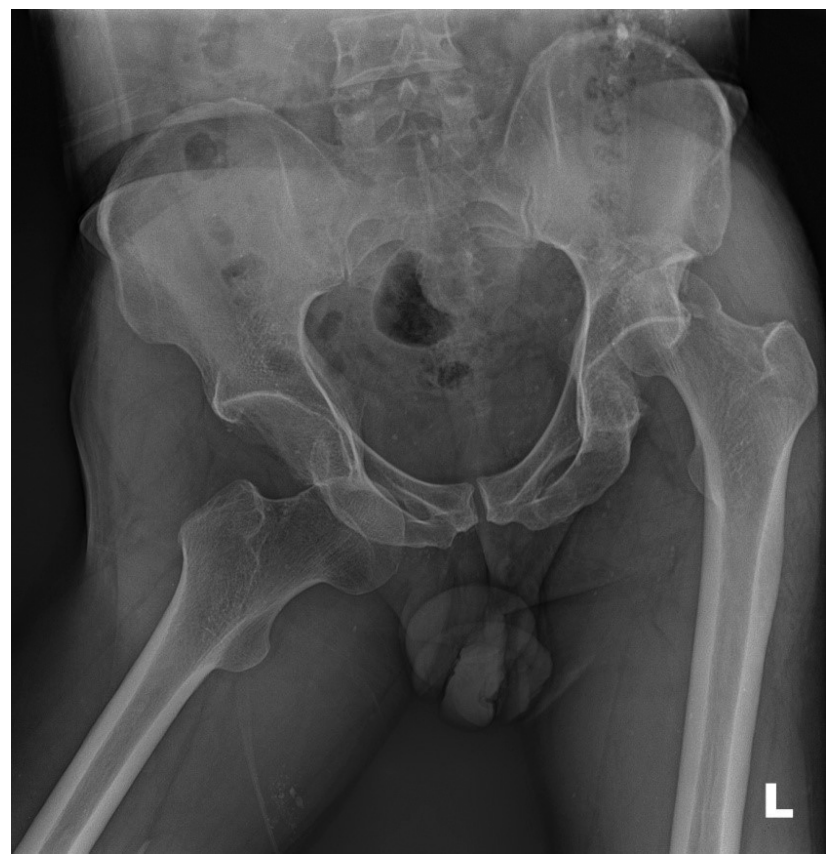

Fig. 1. Initial both hip antero-posterior view of 54 years old male patient rolling several times after falling down from 2 meter height.
The initial radiological test showed an anteroinferiorly dislocated right hip joint and posterosuperiorly dislocated left hip joint with posterosuperior wall fracture of left acetabulum (Fig. 1). As soon as we checked the appearance of dislocation, we put the patient under anesthesia. We manually reducted the posteriorly dislocated left hip joint by Allis method and reducted the anteriorly dislocated right hip joint with addition of an external traction to the proximal femur (Fig. 2). After that, we took a 3 dimension computed tomography (3D-CT) scan in order to identify the condition of both hip joints and location of splinters (Fig. 3). Even though both hip joints were successfully reducted, we decided to conduct an operation because a fracture of the left posterosuperior acetabulum was seen and the size and location of splinters could possibly cause instability of hip joint in the future (Fig. 4). In fluoroscopy during operation, the left hip joint was posteriorly dislocated when we gave flexed, abducted and internally rotated force. We did an open reduction with internal fixation on posterior wall of left acetabulum to prevent future dislocation. We used a posterolateral approach and the short external rotator was intact. The main splinter was fixed with two cortical screws considering the suitability of articular surface.

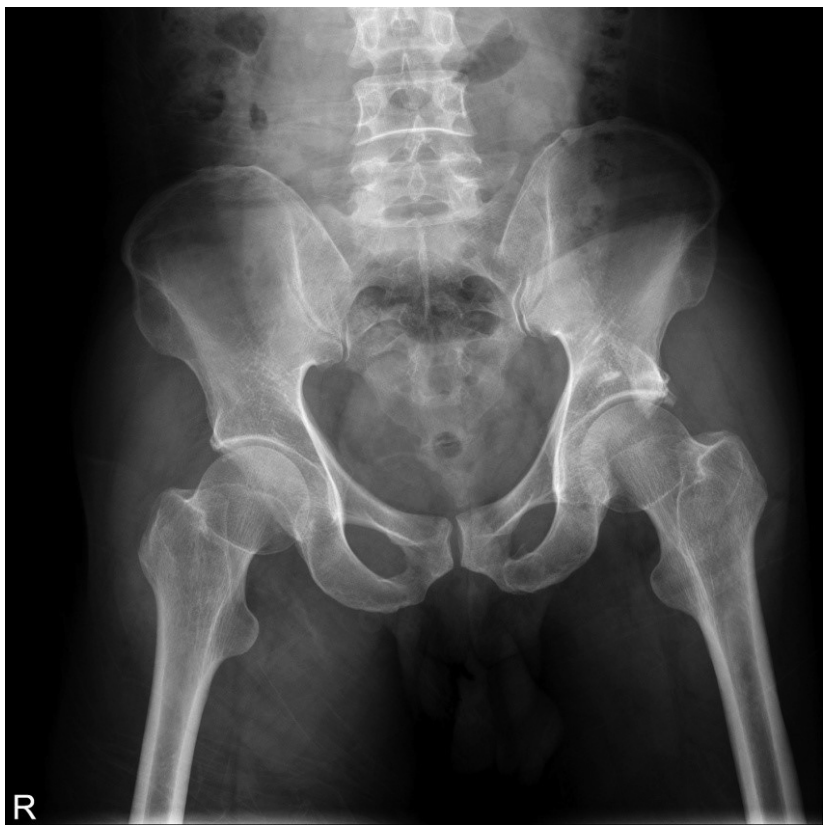

Fig. 2. After reduction with heavy sedation in the emergency department, radiography show concentrically reducted both hip joints with fracture in the postero-superior wall of left acetabulum. 
We carried out buttress plating by putting 8 -hole 3.5 $\mathrm{mm}$ pelvic reconstruction plate from proximal part of ilium to distal part of ischium and fixed it with 6 cancellous screws (Fig. 5). We double checked the absence of an intra-articular fragment. A concentric reduction was possible to make an articular step-off less than $1 \mathrm{~mm}$. 3D-CT scan was done to check joint congruency on the 7th day after operation (Fig. 6).

After the operation, the patient had 4 weeks bed rest with skin traction on both legs. Continuous passive mo-
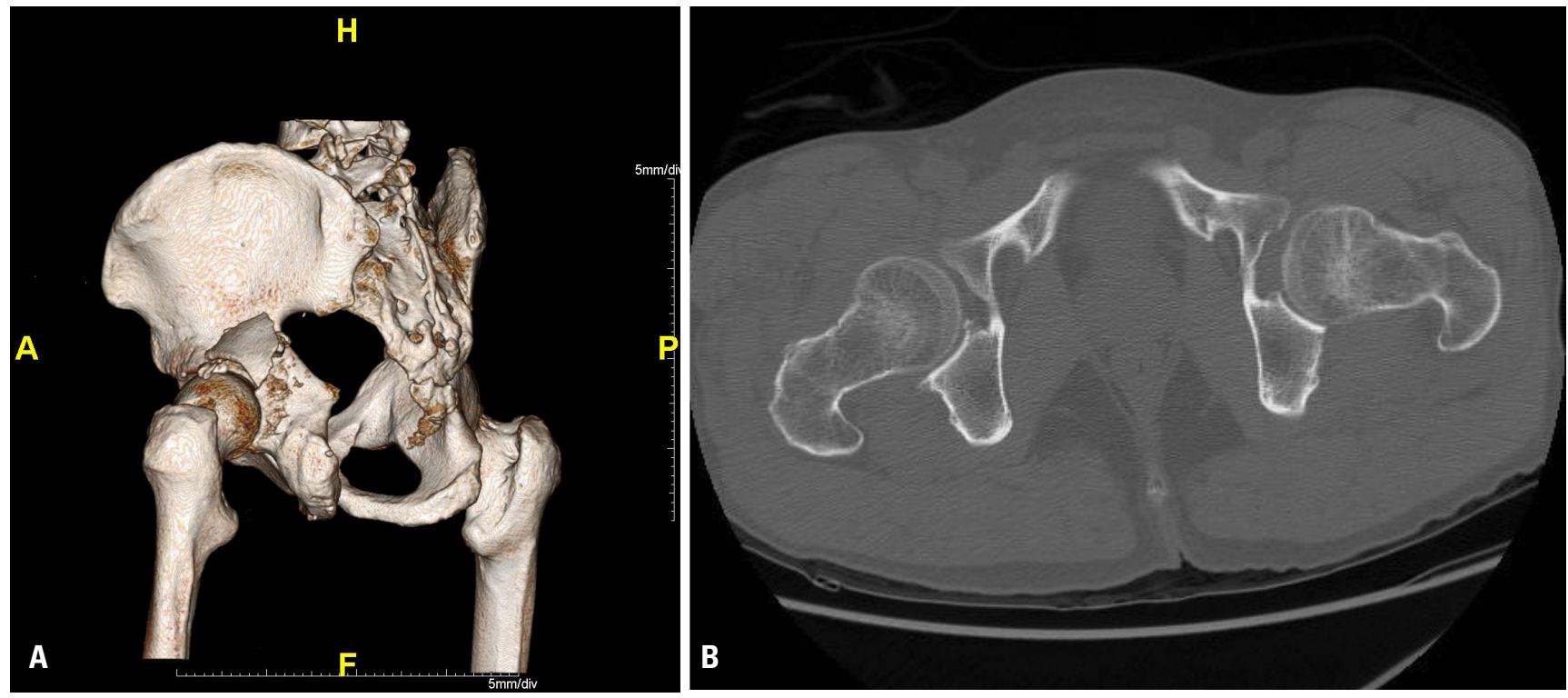

Fig. 3. 3 dimension (3D)-computed tomography with 3D reconstruction image (A) and axial scan (B) after reduction show concentric reduction without any bony fragment in the joint cavity.
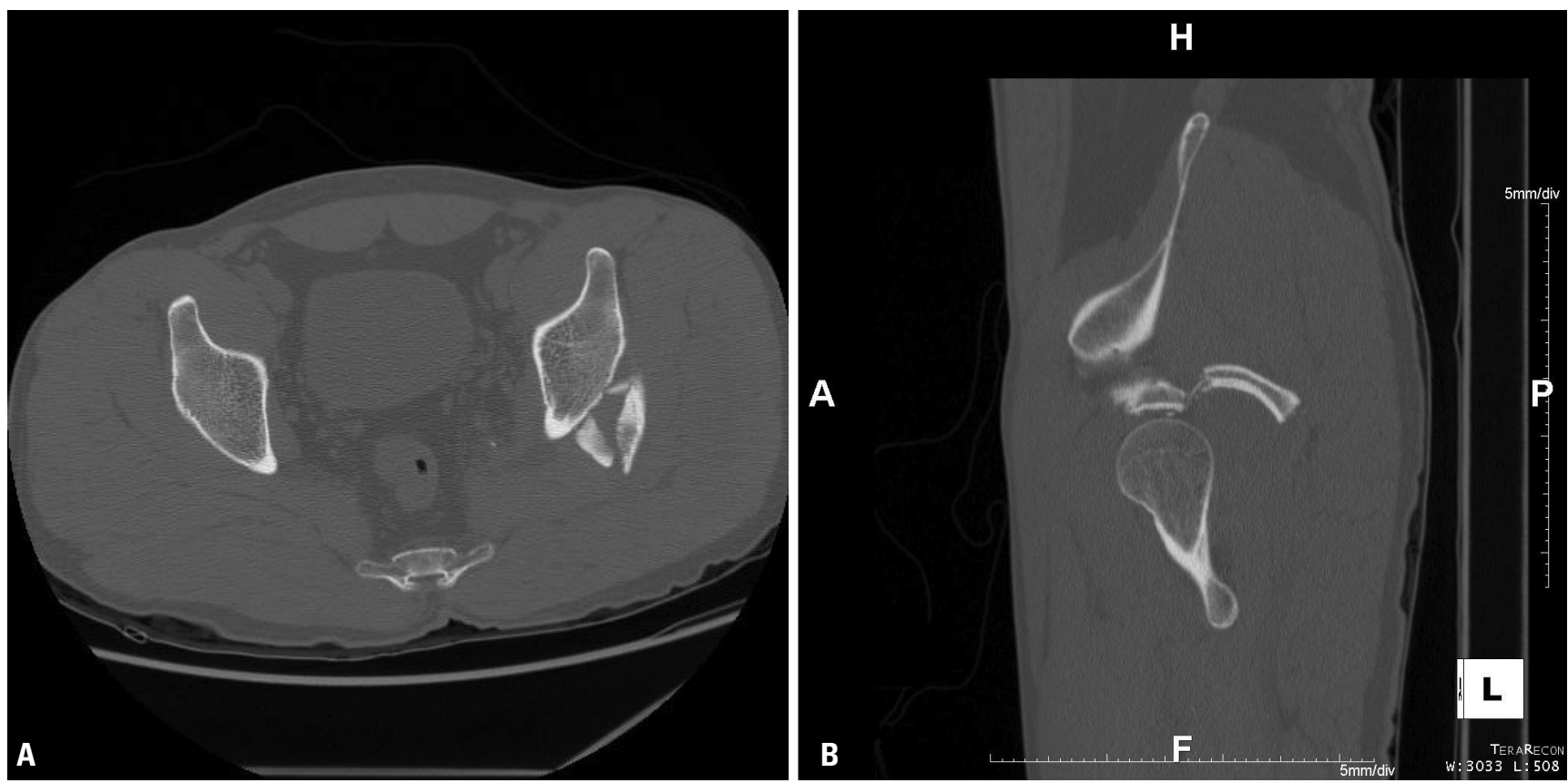

Fig. 4. 3 dimension-computed tomography scan with axial (A) and sagittal (B) views show fracture at the postero-superior aspect of left acetabulum. 

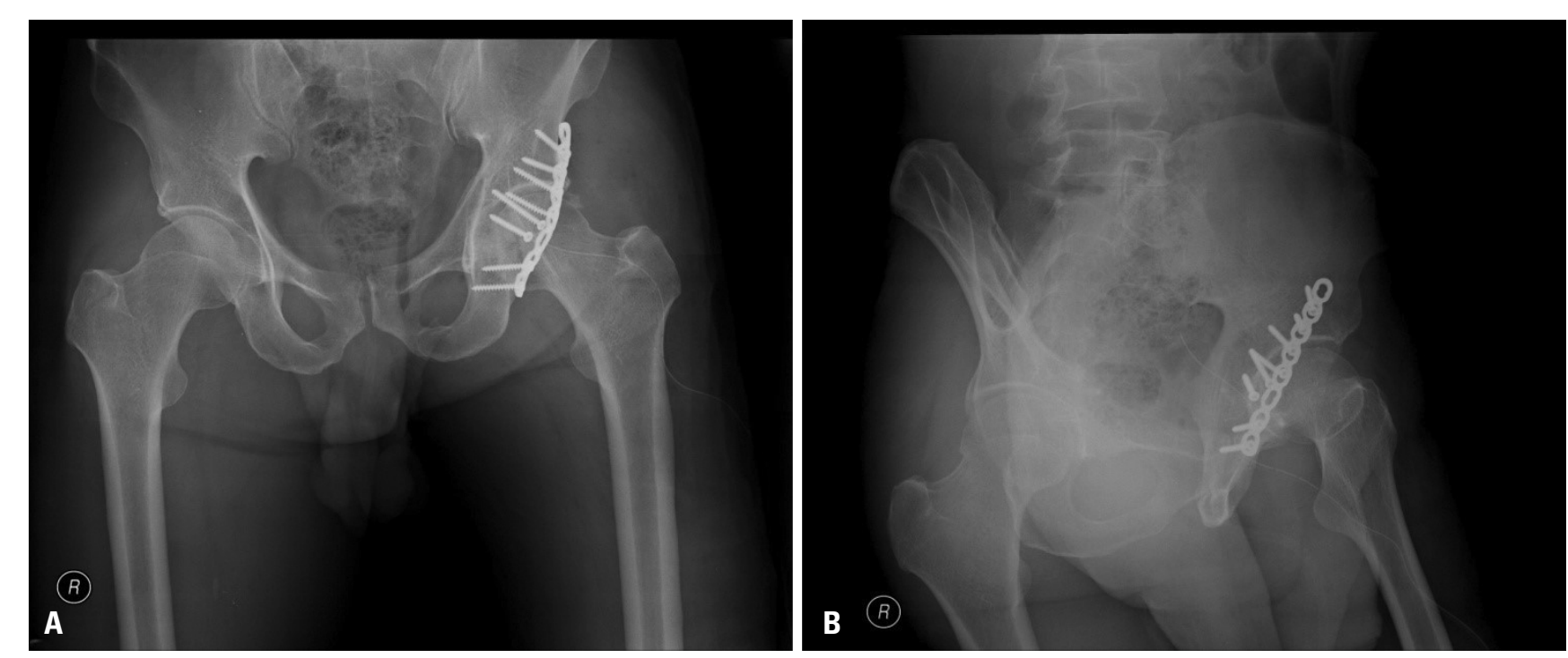

Fig. 5. Radiographies after open reduction and internal fixation of left acetabulum. An 8-hole $3.5 \mathrm{~mm}$ pelvic reconstruction plate was used (A) pelvis A-P view, (B) pelvis oblique view.
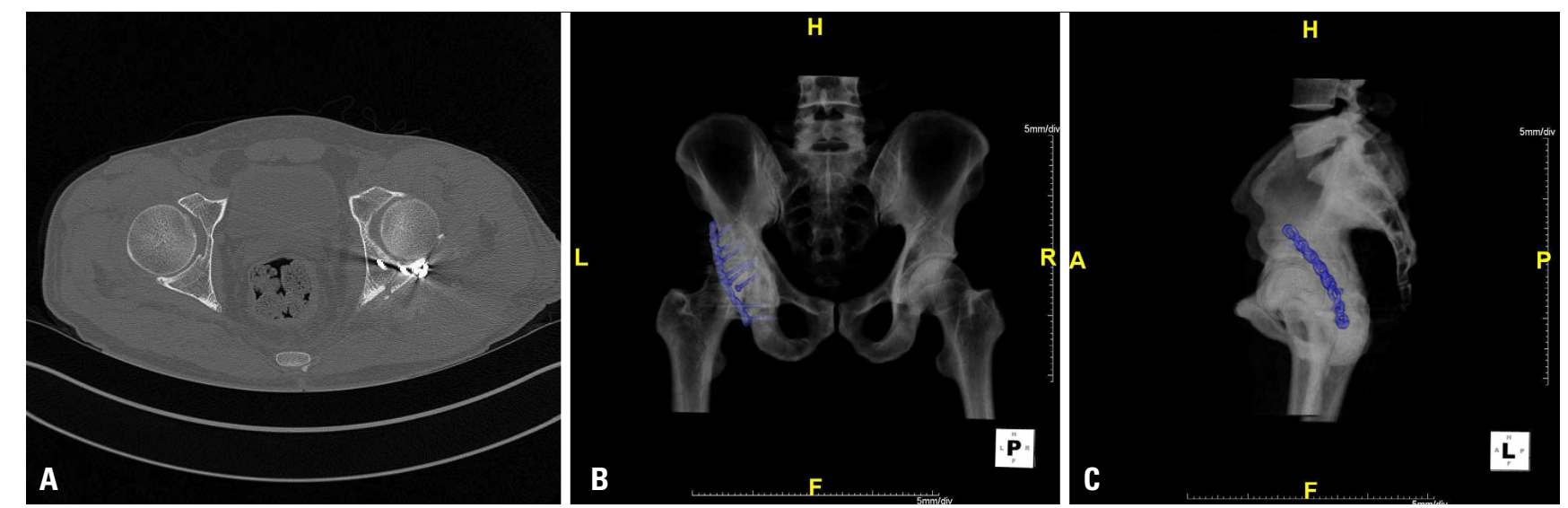

Fig. 6. 3 dimension computed tomography scan on 7 days after operation with axial (A), and bone and metal maximum intensity projection reconstruction image $(B \& C)$ show a well maintained fixation.

tion was done gradually to prevent a limited range of movement in joint. Crutch gait was started from 6 weeks after operation and weight bearing exercise was permitted from 12 weeks after operation. We did not apply radiation treatment or indomethacin to prevent ectopic ossification but wore compression stocking to the patient for preventing deep vein thrombosis. After 24 months of admission, the patient came back to normal life and felt little discomfort with strenuous exercise. There was no sign of avascular necrosis in X-ray and bone scan. Magnetic resonance imaging (MRI) evaluation was not possible due to artifacts of internal fixatives.

\section{Case 2}

A 36 years old male patient got injury when he was in back seat of car which had been turned over. Consciousness of the patient was fine based on the 15 GCS score at time of admission and multiple abrasions were observed on limbs and chest. The right leg was in abducted, externally rotated and flexed position. The left leg was in adducted, internally rotated and flexed position. Also shortness of left leg was seen with naked eyes. We couldn't find other organ trauma or an abnormal finding with hemodynamic stability.

In the physical examination, blood flow of both distal 
legs was normal but the patient complained of general paresthesia of the right leg. The muscle strength of tibialis anterior was checked grade 2-3 and that of both hallucis tendon and flexor digitorum were assessed grade 5. An initial radiological test showed that right hip joint was dislocated anteroinferiorly and left hip joint was dislocated posterosuperiorly with fracture of the posterosuperior wall of left acetabulum. Both legs were manually reducted by Allis method at the ER. We used muscle relaxants and intravenous anesthesia instead of proper anesthesia due to shortness of nil per os time (Fig. 7). The anteriorly dislocated right hip joint was reducted with addition of external traction to the proximal femur. After that, we took 3D-CT scan to identify the condition of both hip joints
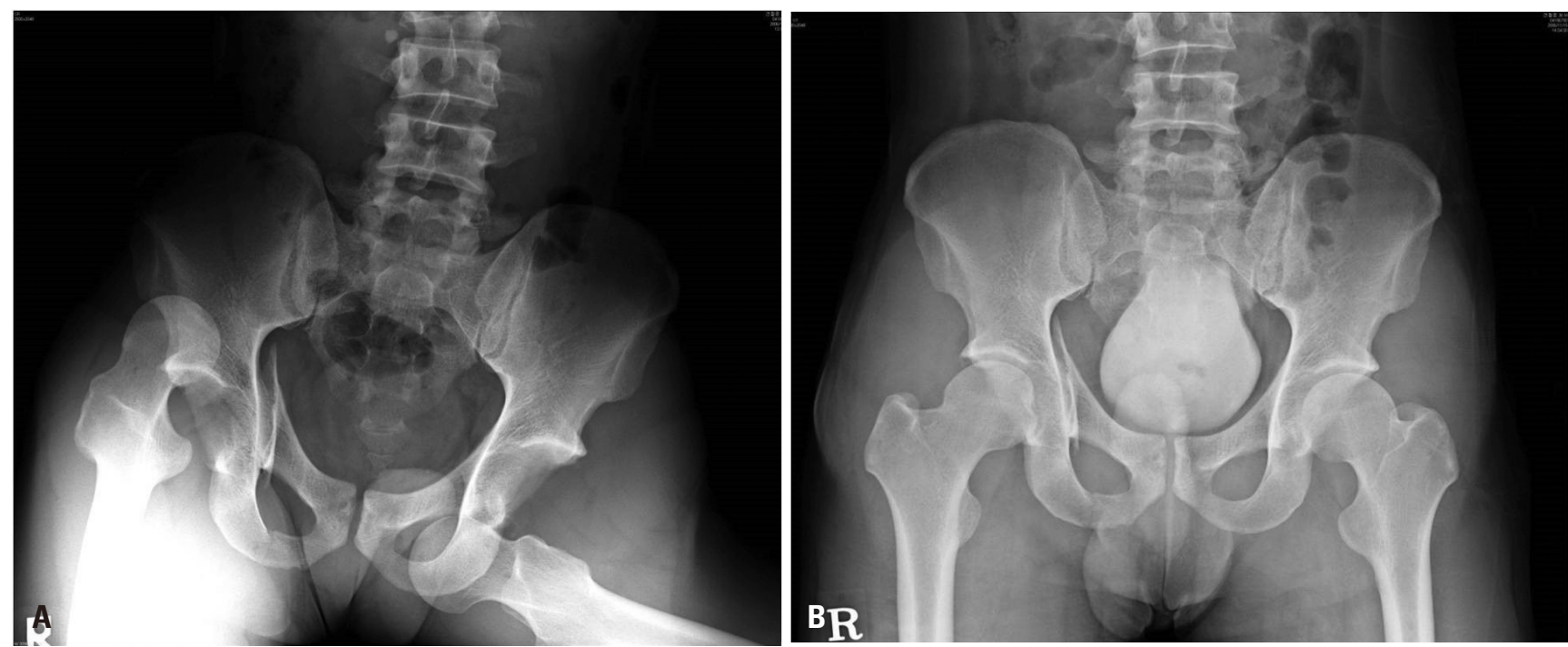

Fig. 7. Initial radiography (A) and radiography after manual reduction (B) of both hip joints of 36 years old male patient after passenger traffic accident. Concentric reduction of both hip joints was done and a fracture of right acetabulum was detected.
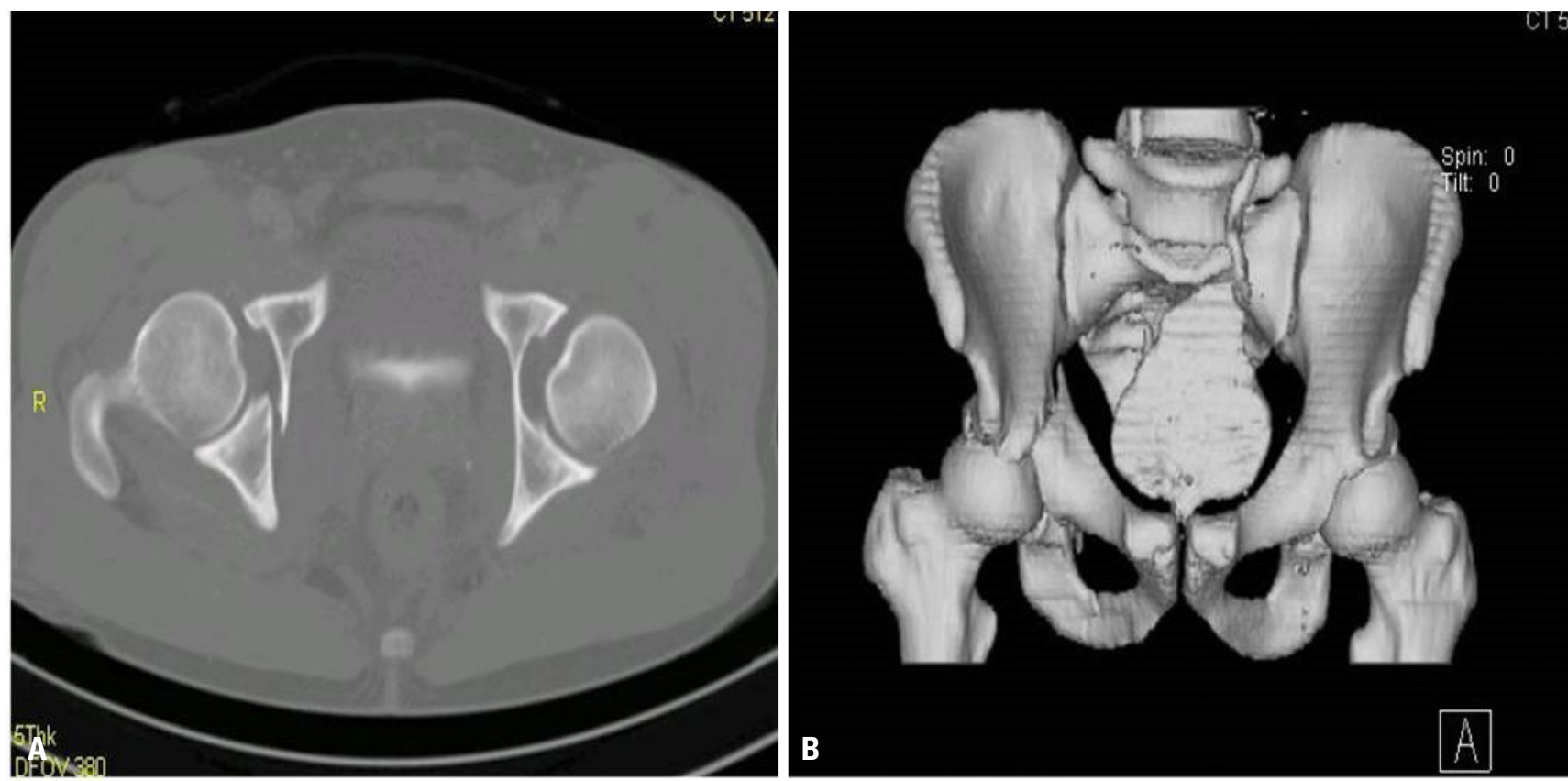

Fig. 8. 3 dimension computed tomography shows fracture of anterior column of pelvis and sacrum axial (A) reconstruction image (B). 


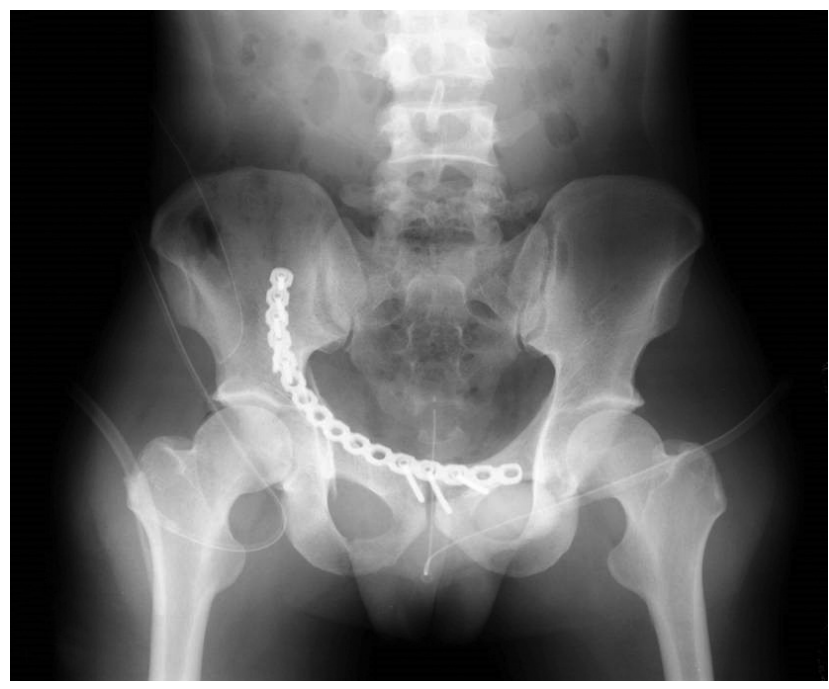

Fig. 9. Radiography after open reduction and internal fixation of right anterior column via ilioinguinal approach.

and location of splinters (Fig. 8). Even though both hip joints were reducted, fracture of sacrum and right anterior column was found. We planned a surgery for the fracture. At the 3rd day of admission, we conducted an open reduction with internal fixation on right anterior column fracture through an ilioinguinal approach (Fig. 9).

After operation, the patient was applied of skin traction for 4 weeks on the right leg and for 2 weeks on the left leg. After that, the patient started active assistive exercise on both legs and hip joints. Crutch walking was permitted from 6 weeks after operation and weight bearing exercise was permitted from 12 weeks after operation. Electromyography (EMG) test was done after 2 weeks of admission and the test results showed a paresthesia due to traumatic sacral nerve injury. 6 months after operation, the patient was permitted active walking. Repeated EMG test was done 4 months later of admission.

We observed a lumbosacral plexopathy between right 5 th lumbar vertebra and 1st sacrum. Also a right lateral femoral cutaneous sensory nerve injury was found. The neurological symptoms of patient improved 6 months later of admission.

Eight months later of hospitalization, the ranges of movement came back to normal on both hip joints. Although paresthesia of right lateral femoral nerve territory and dorsal area of foot remained, dorsiflexion strength of right ankle marked grade 5 . After 18 months, the patient came back to normal life with little a sensory discomfort. There was no sign of avascular necrosis in X-ray and bone scan.

\section{DISCUSSION}

Bilateral dislocation of hip joint is an injury by high energy damage, which could be accompanied by a fracture of acetabulum as well as by a fracture of ipsilateral head, neck, trochanter or shaft of femur. If the damage is relatively low, only ligamentous dislocation could be occurred, but this is a very rare condition including the case report by Kanojia et al. [3]. And cases accompanied by unilateral fracture of acetabulum were no more reported after the cases reported by Martínez et al. [4] and 2 others $[5,6]$. So with this report, we would like to discuss a literature review about the mechanism of injury and treatment as well as about the prognosis.

Most hip joint dislocation are caused by high energy trauma such as traffic accident, falling accident and others [2] The mechanism of the injury is by high speed impact and deceleration force delivered to the lower extremity $[1,2,7]$. If the hip joint is flexed more than 90 degrees, a posteroinferior dislocation and fracture of the posteroinferior wall of acetabulum is occurred. If the impact is applied to the knee joint when the hip joint is abducted and externally rotated, the head of femur will be dislocated anteriorly and a fracture of the anterior wall of acetabulum could be accompanied. If the hip joint is flexed, an anteroinferior dislocation of the head of femur will occur and an anterosuperior dislocation will occur if the hip joint is extended [4]. The injury of knee joint such as fracture of patella is commonly accompanied, but in the cases above there was no knee joint injury found on physical examination and bone scan.

In case 1 , the patient did not remember the pose when injured, but according to the early patterns of the injury, we assumed that the patient might have fallen down with the left knee joint flexed, hip joint adducted and flexed state. A large force was delivered to the left knee joint when he reached the ground, so a posterior dislocation of the hip joint and a fracture of the posterior wall of acetabulum were caused. A relatively small force was delivered to the abducted and extended right knee joint, so an 
anterior dislocation of the right hip joint was caused. The abrasions were focused on the left side so the direction of force could be expected, but the patient stated that he rolled over a sloped rock and it cannot be concluded that the dislocations of both sides were caused by the same force and at the same time. So the possibility cannot be excluded that sequential forces were delivered to both side hip joints.

A fast reposition under intense sedation or general anesthesia must be done within 6 hours after the injury because of many complications of a dislocation of hip joint [8]. Especially in case of anterior dislocation, the dislocated head of femur could be inserted between muscles and become a state of 'locking' at obturator dislocation subtype. A surgical reposition must be done by anterior approach in this case [9].

A skin/skeletal traction is recommended to be applied to 3-8 weeks after reposition $[5,7]$. At our hospital, maintenance of reposition was done by skin traction after an early reposition and if the risk of recurrence was low. The most common long term complication is traumatic arthritis and it occurred in $24 \%$ of patients without an accompanied injury of acetabulum $[4,9,10]$. It rises to $88 \%$ when accompanied with severe injury of acetabulum [1113]. During 18 months Radiologic examinations must be checked every 3 month for an early detection $[1,9]$. An avascular necrosis of the femoral head occurs in $8-15 \%$ of cases with non-invasive reposition and in up to $40 \%$ with invasive reposition until 2 years after surgery [7].

Judet view and lateral view are important to early find an acetabular fracture that could disturb concentric reposition and also when hip joint is posteriorly dislocated attention is required because $\mathrm{T}$ type fracture of the acetabulum could be accompanied [14]. CT scan must be done immediately after the reposition of the hip joint to check bone splinters inside the joint cavity, patterns of the acetabular fracture, accuracy of the reposition and others, and an ebonation or invasive reposition can be planned additionally. Especially splinters of a bone inside the joint cavity must be removed surgically to prevent arthritis [9]. MRI is the most accurate and valuable modality for an avascular necrosis of femoral neck [1], but in cases above, internal fixation was not removed and so bone scan was done instead of MRI. Moreover, a sciatic nerve injury can occur in $7-19 \%$ of cases with posterior dislocation [6] and the femoral neurovascular bundle is rarely injured in cases of anterior dislocation. At an early stage it could be occur because of compression by the dislocated femoral head, but also because of heterotopic ossification or post-operative scar after reposition [15]. Detection by initial physical examination is important and there could be complications such as heterotopic ossification, decrease of the hip joint motion range, deep vein thrombosis and others [9].

Bilateral asymmetric dislocation of hip joint accompanied by unilateral fracture of acetabulum is a very rare case, so statistical verification cannot be done. However, close examinations are required at early treatment and follow-up period because the patterns of dislocation and fracture of hip joint are variable, an orthopedic emergent condition and several complications may occur at both hip joints.

\section{REFERENCES}

1. Agarwal S, Singh GK, Jain UK, Jyoti G. Simultaneous anterior and posterior traumatic dislocation of the hip. A case report with review of the literature. Arch Orthop Trauma Surg 2000;120:236-8.

2. Loupasis G, Morris EW. Asymmetric bilateral traumatic hip dislocation. Arch Orthop Trauma Surg 1998;118:179-80.

3. Kanojia RK, Patra SR, Gupta S. Bilateral asymmetric dislocations of hip joints: an unusual mechanism of injury. Case Rep Orthop 2013;2013:694359.

4. Martínez AA, Gracia F, Rodrigo J. Asymmetrical bilateral traumatic hip dislocation with ipsilateral acetabular fracture. J Orthop Sci 2000;5:307-9.

5. Kaleli T, Alyüz N. Bilateral traumatic dislocation of the hip: simultaneously one hip anterior and the other posterior. Arch Orthop Trauma Surg 1998;117:479-80.

6. Shukla PC, Cooke SE, Pollack CV Jr, Kolb JC. Simultaneous asymmetric bilateral traumatic hip dislocation. Ann Emerg Med 1993;22:1768-71.

7. Lam F, Walczak J, Franklin A. Traumatic asymmetrical bilateral hip dislocation in an adult. Emerg Med J 2001;18:506-7.

8. Hougaard K, Thomsen PB. Coxarthrosis following traumatic posterior dislocation of the hip. J Bone Joint Surg Am 1987;69:679-83. 
9. Dudkiewicz I, Salai M, Horowitz S, Chechik A. Bilateral asymmetric traumatic dislocation of the hip joints. J Trauma 2000;49:336-8.

10. Sraj SA, Lakkis S. Simultaneous anterior and posterior traumatic hip dislocation: a case report and review of literature. Injury Extr 2007;38:327-33.

11. Upadhyay SS, Moulton A, Srikrishnamurthy K. An analysis of the late effects of traumatic posterior dislocation of the hip without fractures. J Bone Joint Surg Br 1983;65:150-2.

12. Upadhyay SS, Moulton A. The long term results of trau- matic posterior dislocation of the hip. J Bone Joint Surg Br 1981;63:548-51.

13. Rodríguez-Merchán EC. Coxarthrosis after traumatic hip dislocation in the adult. Clin Orthop Relat Res 2000;377:92-9.

14. Alonso JE, Volgas DA, Giordano V, Stannard JP. A review of the treatment of hip dislocations associated with acetabular fractures. Clin Orthop Relat Res 2000;377:32-43.

15. Cornwall R, Radomisli TE. Nerve injury in traumatic dislocation of the hip. Clin Orthop Relat Res 2000;(377):84-91. 